\title{
MIST1 regulates SNAI1 and acts through the PTEN/AKT signaling axis to promote anoikis resistance in human melanoma cells
}

\author{
YIJU LEE $^{1 *}$, WEIFENG YAO ${ }^{2 *}$, CHUNJUN YANG $^{1}$, YUNRUI LI $^{3}$, \\ HAIYANG NI $^{2}$, LEI WANG ${ }^{2}$, BIN JI $^{2}$, YONGGE GU ${ }^{2}$ and SEN YANG ${ }^{1}$ \\ ${ }^{1}$ Department of Dermatology, The First Affiliated Hospital of Anhui Medical University, \\ Hefei, Anhui 230022; ${ }^{2}$ Department of Dermatology, Tianjin Academy of Traditional Chinese Medicine Affiliated Hospital, \\ Tianjin 300120; ${ }^{3}$ School of Basic Medicine, Tianjin Medical University, Tianjin 300070, P.R. China
}

Received November 10, 2016; Accepted June 23, 2017

DOI: $10.3892 /$ etm.2018.6225

\begin{abstract}
Cutaneous malignant melanoma (CMM) is one of the most dangerous types of skin cancer. The prognosis of CMM patients with ulcers, regional lymph node metastasis or organ metastasis is poor. In this process, resistance to anoikis is a critical step in tumor cell metastasis. Tumor cells survive in the vascular and lymphatic system through the escape of anoikis to finally form clones in the distal tissue. The present study revealed that muscle intestine and stomach expression 1 (MIST1), a secreting cell-restricted transcription factor, was overexpressed in melanoma cells. At the same time, the expression of SNAI1 was also high. High expression of MIST1 and SNAI1 all contributed to melanoma cells bypassing anoikis. By changing the expression of MIST1, SNAI1 was indicated to be a downstream gene of MIST1. Chromatin immunoprecipitation and luciferase reporter gene technology revealed that MIST1 promoted the expression of SNAI1 by directly binding to its promoter region. Furthermore, inhibition of the phosphorylation/activity of Akt by LY294002 and knockdown of phosphatase and tensin homologue (PTEN) with simultaneous upregulation or knockdown of MIST1 revealed that SNAI1 improved the phosphorylation of Akt by inhibiting the expression of PTEN. These results suggested that MIST1 hijacked the PTEN/AKT signaling pathway through directly regulating SNAI1 and affected the anoikis resistance capacity of melanoma cells.
\end{abstract}

Correspondence to: Professor Sen Yang, Department of Dermatology, The First Affiliated Hospital of Anhui Medical University, 218 Jixi Road, Hefei, Anhui 230022, P.R. China

E-mail: sen_yangaca@outlook.com

${ }^{*}$ Contributed equally

Key words: melanoma, anoikis, muscle intestine and stomach expression 1, phosphatase and tensin homologue, AKT, transcriptional regulation

\section{Introduction}

Cutaneous malignant melanoma (CMM) is a type of skin tumor. This malignant type of neoplasm evolves from melanoma cells at the base of the epidermis. Its incidence rate accounts for $1-3 \%$ of all malignant tumor types and 5-10\% of malignant skin tumors, and has demonstrated a clear upward trend in recent years (1). It is currently thought that the pathogenesis of MM is primarily associated with solar irradiation of the skin. Ultraviolet rays in the sunlight burn the skin to induce DNA mutations. Mutations in P16 or cyclin-dependent kinase inhibitor 2A located on the short arm of chromosome 9 have been identified as the major cause of genetic pre-disposition to MM (2). Surgery, radiotherapy and chemotherapy are the major treatments of MM; however, the efficacy is low. The discovery of melanoma-associated mutations in genes in recent years and in-depth study may provide approaches for targeted therapies for a new era of MM treatment.

Normal cells gather together and adhere to the extracellular matrix (ECM). They survive through mutual substances and signal conduction. Once they lose contact with the ECM, the cells undergo programmed death, also known as apoptosis. This form of cell death was first named anoikis in 1994 (3). Anoikis is indispensable to maintain the stability of the body tissue. Its main role is to prevent abnormal cell growth or adhesion of cells to the abnormal ECM. Resistance to anoikis is a hallmark of tumor metastasis, allowing tumor cells to spread through the circulatory system to other distant organs. Tumor cells are resistant to apoptosis through autocrine and paracrine mechanisms after losing cell-to-ECM and cell-to-cell contact, and regain the ability to diffuse, metastasize and invade (4-6). Studies have demonstrated that integrins as inhibitors of anoikis have a profound effect on cell survival $(7,8)$. The phosphoinositide 3-kinase (PI3K)/Akt and mitogen-associated protein kinase kinase/extracellular signal-regulated kinase pathways are involved in the critical step of resistance to anoikis (4).

Muscle intestine and stomach expression 1 (MIST1), also referred to as basic helix-loop-helix (bHLH) family, 
member a15, is a transcriptional factor. The bHLH motif, which is consistent throughout this family, contains $\sim 60$ amino acids and consists of a basic region and a HLH motif that binds to DNA (9). It has been reported that MIST1 has an important role in maintaining normal cell numbers and promoting terminal differentiation of acinar cells in pancreatic exocrine glands. These effects indicate that MIST1 is a key anti-cancer factor in pancreatic tumorigenesis (10).

The transcription factor SNAI1 is involved not only in the regulation of primordial germ cell migration, gastrulation and neural crest migration during normal embryonic development, but also has an important role in the occurrence, recurrence, invasion and metastasis of organ tumors and organ fibrosis (11-13). SNAI1 inhibits the expression of E-cadherin and has a crucial role in epithelial-to-mesenchymal transition (EMT) $(14,15)$. Interfering with SNAI1 expression may increase the death rate of cancer cells (16). In addition, SNAI1 inhibits the expression of phosphatase and tensin homologue (PTEN) through binding to the promoter of PTEN on chromosome 10. This leads to resistance to gamma-ray-induced apoptosis (17).

$\mathrm{PI} 3 \mathrm{~K} / \mathrm{AKT}$ is an important signaling pathway that facilitates the immortalization of cells and promotes the development of normal vascular and tumor angiogenesis (18-21). PTEN is a lipid phosphatase that dephosphorylates phosphatidylinositol $(3,4,5)$-trisphosphate and blocks the PI3K/AKT pathway. The effect of PTEN on p27 appears to be mediated via inhibition of the PI3K/Akt pathway. After the expression of PTEN is inhibited, cell cycle progression is accelerated through phosphorylation of Akt and glycogen synthase kinase-3 (22). It is reported that SNAI1 directly represses PTEN and leads to activation of the PI3K/Akt pathway, and then leads to inactivation and phosphorylation of the pro-apoptotic protein Bcl-2-associated death promoter. This contributes to anoikis resistance (23).

The present study initially explored the association between MIST1 and SNAI1 in human melanoma cells. It was found that MIST1 and SNAI1 contributed to the detachment of cells from the cell matrix by altering the expression of certain proteins. Their upregulation was beneficial for survival after suspension culture. The expression of SNAI1 changed with the alteration of MIST1. It was demonstrated that the PTEN/AKT signaling pathway also participated in their function; SNAI1 was found to inhibit the expression of PTEN and activate Akt. This helped cells to bypass anoikis. MIST1 was identified bind to the promoter of SNAI1 and activate the expression of SNAIL1 as a transcription factor, as indicated using chromatin immunoprecipitation (ChIP) and luciferase reporter gene technology.

\section{Materials and methods}

Cell culture. HUVEC, NHEM 2493, A375 and MV3 cell lines were obtained from the American Type Culture Collection (ATCC; Manassas, VA, USA). HUVEC was maintained in endothelial cell growth-2 medium supplemented with $2 \%$ fetal bovine serum, $15 \mathrm{ng} / \mathrm{ml}$ insulin-like growth factor-1, $5 \mathrm{ng} / \mathrm{ml}$ epidermal growth factor, 0.75 Units $/ \mathrm{ml}$ heparin sulfate, $5 \mathrm{ng} / \mathrm{ml}$ vascular endothelial growth factor, $5 \mathrm{ng} / \mathrm{ml}$ basic fibroblast growth factor, $10 \mathrm{mM} \mathrm{L}$-glutamine, $1 \mathrm{ng} / \mathrm{ml}$ hydrocortisone hemisuccinate and $50 \mu \mathrm{g} / \mathrm{ml}$ ascorbic acid (all from Lonza Group, Ltd., Basel, Switzerland). NHEM was maintained in serum- and phorbol myristate acetate-free melanocyte growth medium M2 (Promocell GmbH, Heidelberg, Germany). MV3 and A375 were maintained in Dulbecco's modified Eagle's medium supplemented with 15\% fetal bovine serum (both from Gibco; Thermo Fisher Scientific, Inc., Waltham, MA, USA). Cells were maintained in an incubator at $37^{\circ} \mathrm{C}$ in a humidified atmosphere containing $5 \% \mathrm{CO}_{2}$.

Adhesion assay. Six-well plates were coated with $10 \mathrm{~g} / \mathrm{ml}$ fibronectin (cat. no. F2006; Sigma Aldrich; Merck KGaA, Darmstadt, Germany). Then they were blocked at room temperature with $0.2 \%$ BSA for $2 \mathrm{~h}$. A total of $1 \times 10^{5}$ cells were placed in each well and incubated in the $37^{\circ} \mathrm{C}$ cell incubator for $30 \mathrm{~min}$. Medium was discarded and excess cells were rinsed using PBS. Adhered cells were stained with crystal violet at room temperature for $10 \mathrm{~min}$ and photographed under a light microscope.

Cell death detection ELISA. A total of $5 \times 10^{4}$ cells were placed in normal and low-attachment surface 24 -well plates. A Cell Death Detection ELISA ${ }^{\text {PLUS }}$ (cat. no. 11774425001; Roche Diagnostics, Basel, Switzerland) was used to detect apoptosis level according to the manufacturer's instructions.

RNA isolation and polymerase chain reaction $(P C R)$ analysis. TRIzol (Invitrogen; Thermo Fisher Scientific, Inc.) was used for extracting total RNA from cells. ReverAid First Strand cDNA Synthesis kit (cat. no. K1622; Fermentas; Thermo Fisher Scientific, Inc.) was applied to reverse-transcribe mRNA with a polyA tail into complementary (c)DNA. PCR Master Mix (cat. no. K0172; Invitrogen; Thermo Fisher Scientific, Inc.) was used for PCR. Cycling conditions for the PCR reaction were as follows: Initial denaturation step of $3 \mathrm{~min}$ at $94^{\circ} \mathrm{C}$, followed by 31 cycles consisting of $30 \mathrm{sec}$ at $94^{\circ} \mathrm{C}, 30 \mathrm{sec}$ at $55^{\circ} \mathrm{C}$ and $30 \mathrm{sec}$ at $72^{\circ} \mathrm{C}$, with a final extension period of $7 \mathrm{~min}$ at $72^{\circ} \mathrm{C}$.

Sequences of the primers were as follows: MIST1 forward, 5'-GCGGATGCACAAGCTAAATAAC-3' and reverse, 5'-CTCGATCTTGGAGAGCTTCTTG-3'; SNAI1 forward, 5'-CCTTCGTCCTTCTCCTCTACTT-3' and reverse, 5'-GGC ACTGGTACTTCTTGACATC-3'; GAPDH forward, 5'-GCG GATGCACAAGCTAAATAAC-3' and reverse, 5'-CTCGAT CTTGGAGAGCTTCTTG-3'.

Protein extraction and western blot analysis. Laemmli sample buffer containing 5\% 2-mercaptoethanol (both from Bio-Rad Laboratories, Inc., Hercules, CA, USA) was used to extract total protein from cells. Pierce ${ }^{\mathrm{TM}}$ BCA Protein assay kit (cat. no. 23227; Invitrogen; Thermo Fisher Scientific, Inc.) was used to detect protein concentration and $20 \mu \mathrm{g}$ protein was loaded in each well. Protein samples were separated by $8-15 \%$ SDS-PAGE, followed by transfer onto a polyvinylidene fluoride membrane (cat. no. PVM020C-160; Pall Life Sciences, Port Washington, NY, USA). The following primary antibodies were used: Rabbit MIST1 monoclonal antibody (mAb) (cat. no. ab180889; 1:2,000 dilution in 5\% w/v milk; Abcam, Cambridge, MA, USA), goat SNAI1 mAb (cat. no. ab53519; 1:2,000 dilution in 5\% w/v milk; Abcam), 
rabbit $\mathrm{Akt} \mathrm{mAb}$ [cat. no. 9272L; 1:2,000 dilution in 3\% w/v bovine serum albumin (BSA); Cell Signaling Technology, Inc., Danvers, MA, USA], rabbit phospho-(p)Akt (Ser473) mAb (cat. no. 4060; 1:1,000 dilution in 3\% w/v BSA; Cell Signaling Technology, Inc.), rabbit PTEN mAb (cat. no. 9188; 1:2,000 dilution in 3\% w/v BSA; Cell Signaling Technology, Inc.) and mouse GAPDH mAb (cat. no. ab8245; 1:5,000 dilution in $5 \% \mathrm{w} / \mathrm{v}$ milk; Abcam). The primary antibodies were incubated at $4^{\circ} \mathrm{C}$ overnight. Goat Anti-Mouse IgG H\&L (cat. no. ab6789; 1:2,000), Goat Anti-Rabbit IgG H\&L (cat. no. ab6721; 1:2,000 dilution in 3\% w/v BSA) and Rabbit Anti-Goat IgG H\&L (cat. no. ab6741; 1:2,000 dilution in 5\% w/v milk; all from Abcam) were used as secondary antibodies. The secondary antibodies were incubated at room temperature for $4 \mathrm{~h}$. Bands were visualized using a Chemiluminescent horseradish peroxidase substrate (cat. no. P90720; EMD Millipore; Billerica, MA, USA) and images were captured using X-OMAT BT Film (cat. no. 6535876; Carestream Health, Inc., Rochester, NY, USA). The concentration of LY294002 (cat. no. 9901; Cell Signaling Technology, Inc., Boston, MA, USA) was $10 \mu \mathrm{M}$ to suppress p-AKT (Ser473).

Construction of overexpression and knockdown vectors. cDNA of HUVEC was used as a template to amplify the full-length open reading frames of MIST1 and SNAI1 by PCR, which were cloned in the expression vector pcDNA3.1 (cat. no. V79020; Invitrogen; Thermo Fisher Scientific, Inc.). pLKO.1 containing small hairpin (sh)RNAs specifically targeting MIST1, SNAI1 and PTEN (Shanghai GeneChem Co., Ltd., Shanghai, China) were used to knockdown these genes in the cell lines. The shRNAs had the following sequences: shRNA-1 targeting MIST1, 5'-CCGGCCAAGGGTCTG CGGAGC-3'; shRNA-2 targeting MIST1, 5'-CCATGTCCA GCAGCCGCCTCC-3'; shRNA-1 targeting SNAI1, 5'-TTTA CCTTCCAGCAGCCCTAC-3'; shRNA-2 targeting SNAI1, 5'-ACCTCAGCCTGGGTGCCCTCA-3'; shRNA-1 targeting PTEN, 5'-AGAGTTGCACAATATCCTTTT-3'; shRNA-2 targeting PTEN, 5'-GAGGAAACCTCAGAAAAAGTA-3'. A $293 \mathrm{~T}$ cell lentiviral packaging system was used. A total of $2.5 \mu \mathrm{g} \mathrm{Rev}, 3 \mu \mathrm{g} \mathrm{Rev}, 3,5 \mu \mathrm{g}$ Rev and $12 \mu \mathrm{g}$ target plasmid were transfected into $293 \mathrm{~T}$ cells in a $10 \mathrm{~cm}$ cell culture dish with polyethyleneimine (cat. no. B600070; ProteinTech Group, Inc., Chicago, IL, USA) at a concentration of $1.7 \mu \mathrm{g} / \mu \mathrm{l}$. After $4 \mathrm{~h}$ of transfection, the medium was changed to complete medium to collect the virus. The collected virus infected the target cells 3 times for $8 \mathrm{~h}$ each.

ChIP assay. Direct combination and transcriptional activation of SNAI1 by MIST1 was predicted by the Encyclopedia of DNA Elements in the UCSC database (http://genome.ucsc.edu/ENCODE/). The JASPAR database (http://jaspar.genereg.net/) was used to predict the MIST1 binding motif. Cells were fixed with $1 \%$ formaldehyde and terminated with $2.5 \mathrm{mM}$ glycine. The scraped cells were sonicated for lysis in PBS with sodium thiosulfate. The lysates were divided into three aliquots, one of which was a positive control and received no treatment and one of which was a negative control, which was incubated with immunoglobulin $G$ (cat. no. I5006) and protein G PLUS-Agarose (cat no. P7700) 9both from Sigma-Aldrich; Merck KGaA). One third of the cell lysate was used as the test group, and was incubated with antibody against MIST1 (1:100) and Protein G PLUS-Agarose. After removal of RNA and protein, DNA was extracted with phenol-chloroform, respectively. Next, the degree of enrichment was detected using real-time quantitative PCR.

Luciferase reporter gene technology. At $24 \mathrm{~h}$ prior to transfection, $1 \times 10^{5}$ A375 and MV3 cells were seeded into 24-well plates. pGL3 luciferase reporters vector containing target fragments ( 800 ng) were complexed with $1 \mu$ l Lipofectamine 2000 (Invitrogen; Thermo Fisher Scientific, Inc.), and then added to the cells. These target fragments included $-1,199$ to -753 , $-2,135$ to $-753,-3,235$ to $-753,-4,024$ to $-753,-2,135$ to $-1,200$, $-3,235$ to $-1,200$ and $-4,024$ to $-1,200$ upstream of TSS site of SNAI1. Renilla reniformis luciferase (pRL-TK) was used as the transfection control. Cells were harvested and lysed $24 \mathrm{~h}$ later. The luciferase activity was measured using a Dual-Luciferase Reporter Assay System in a Promega GloMax 20/20 Luminometer (Promega Corp., Madison, WI, USA).

Statistical analysis. Statistical analyses were performed using SPSS 19.0 software (SPSS Inc., Chicago, IL, USA). Data are presented as the mean \pm standard deviation for continuous data. One-way analysis of variance was performed for comparison analysis. Dunnett's test was used for pairwise comparisons of multiple treatment groups. All experimental groups were compared with the control groups. $\mathrm{P}<0.05$ was considered to indicate a statistically significant difference.

\section{Results}

Expression of MIST1 positively correlates with SNAII in normal and melanoma cells. MIST1 is the first transcription factor identified as a protein specifically expressed in serous exocrine cells (24). MIST1 was identified to have an important role in pancreatic cancer. SNAI1 is involved in EMT and is closely associated with tumor metastasis, recurrence and prognosis as a classic zinc finger protein. However, the interaction between these two proteins and their function in human melanoma cells has remained to be fully elucidated. The present study found that the expression of MIST1 and SNAI1 was upregulated in the human melanoma cell lines A375 and MV3 at the RNA and protein level (Fig. 1A and B). This indicated that MIST1 and SNAI1 are likely to have a role in the development and progression of melanoma. Furthermore, the expression of MIST1 and SNAI1 in normal or melanoma cells was substantially increased after culture in a 24-well plate with a low attachment surface for $24 \mathrm{~h}$ (Fig. 1C and D). Leaving the matrix stimulated the cells to express MIST1 and SNAI1. These results indicated that MIST1 and SNAI1 may help cells to bypass anoikis after loss of anchorage to their physical environment.

MIST1 and SNAI1 disrupt cell-matrix adhesion and promote anchorage independence. Previous studies have reported that SNAI1 has a pro-apoptotic function and helps cells to bypass anoikis (23). However, the association of MIST1 with anoikis has remained elusive.

To overexpress MIST1 and SNAI1, a 293 T cell lentiviral packaging system was used, with which HUVEC and 
A

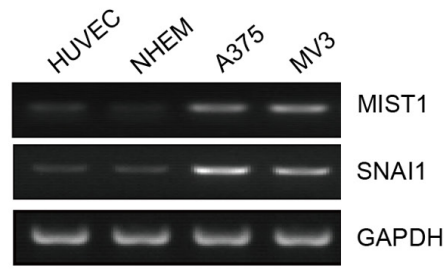

C

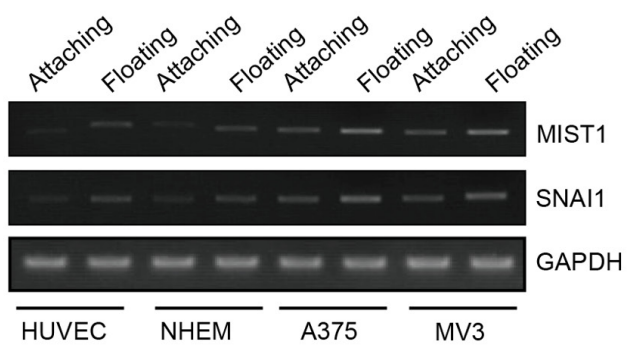

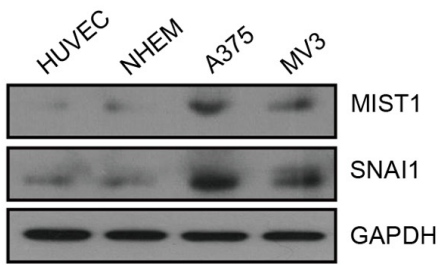

D

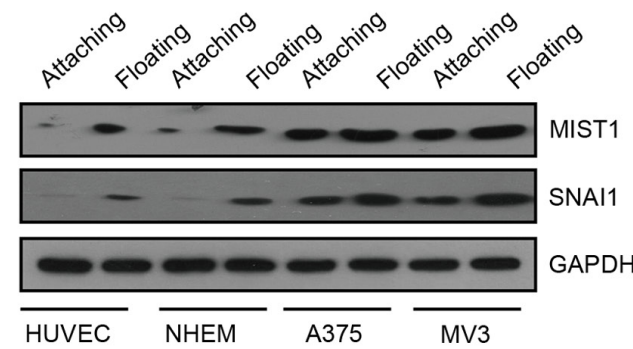

Figure 1. Expression of MIST1 is positively associated with SNAI1 in human normal and melanoma cells. (A and B) RNA and protein levels of MIST1 and SNAI1 in HUVEC, NHEM 2493, A375 and MV3 cell lines. (C and D) RNA and protein levels of MIST1 and SNAI1 in attached and floating cell lines. MIST1, muscle intestine and stomach expression 1; HUVEC, human umbilical vein endothelial cells; NHEM 2493, normal human epidermal melanocytes.

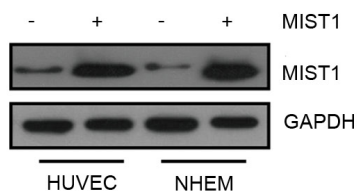

D

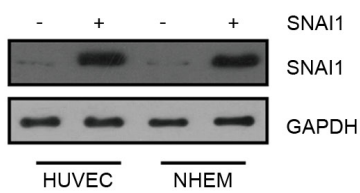

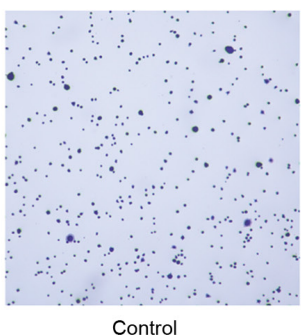

$E$

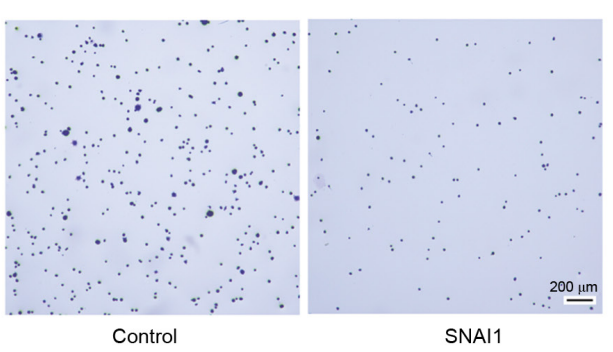

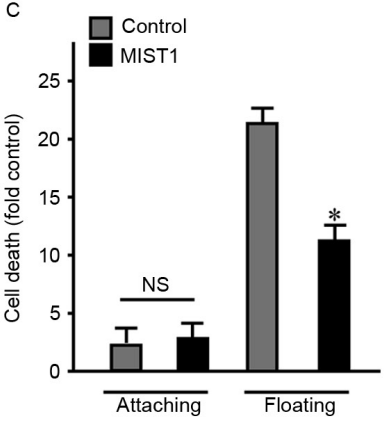

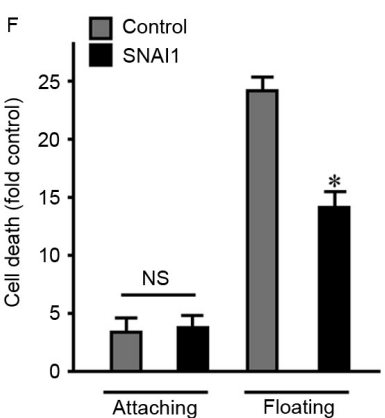

Figure 2. MIST1 and SNAI1 disrupt cell-matrix adhesion and promote anchorage independence. (A) MIST1 was ectopically expressed in HUVEC and NHEM 2493 cell lines and confirmed by western blot analysis. (B) Overexpression of MIST1 reduced the adhesion ability of HUVEC (scale bar, $200 \mu \mathrm{m}$ ). (C) MIST1 enhanced the viability of HUVEC in suspension culture, indicating bypassing of anoikis. (D) SNAI1 was ectopically expressed in HUVEC and NHEM 2493 cell lines and confirmed by western blot analysis. (E) Overexpression of SNAI1 reduced the adhesion ability of HUVEC (scale bar, $200 \mu \mathrm{m}$ ). (F) SNAI1 enhanced the viability of HUVEC in suspension culture, indicating bypassing of anoikis. Findings for NHEM 2493 were similar, data not shown. NS, no significance; ${ }^{*} \mathrm{P}<0.05$ vs. control. MIST1, muscle intestine and stomach expression 1; HUVEC, human umbilical vein endothelial cells; NHEM 2493, normal human epidermal melanocytes.

NHEM were transfected to endogenously express MIST1 and SNAI1 (Fig. 2). First, overexpression of MIST1 was confirmed by western blot analysis (Fig. 2A). Subsequent cell behavioral experiments revealed that MIST1 expression resulted in a decreased adherence of normal human HUVEC and NHEM to fibronectin (Fig. 2B). As anoikis-sensitive cells, HUVEC and NHEM were used to assess the influence of MIST1 on anoikis. The ratio of dead cells in HUVEC and
NHEM overexpressing MIST1 was detected after floating for $24 \mathrm{~h}$ by using a BD Cell Death Detection ELISA kit. After overexpression of MIST1, resistance to anoikis was obviously improved (Fig. 2C). Furthermore, HUVEC and NHEM were transfected with SNAI1 and subjected to the above experiments, revealing a similar effect of SNAI1 to that of MIST1 (Fig. 2D-F). These results indicated that MIST1 and SNAI1 promoted anchorage independence in normal human cells. 

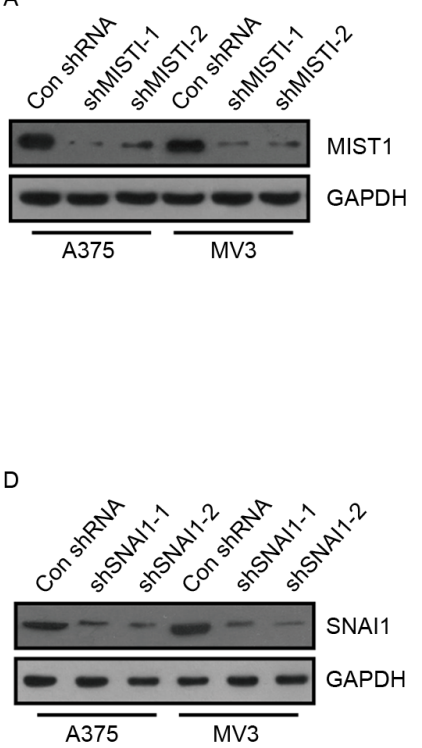

$B$
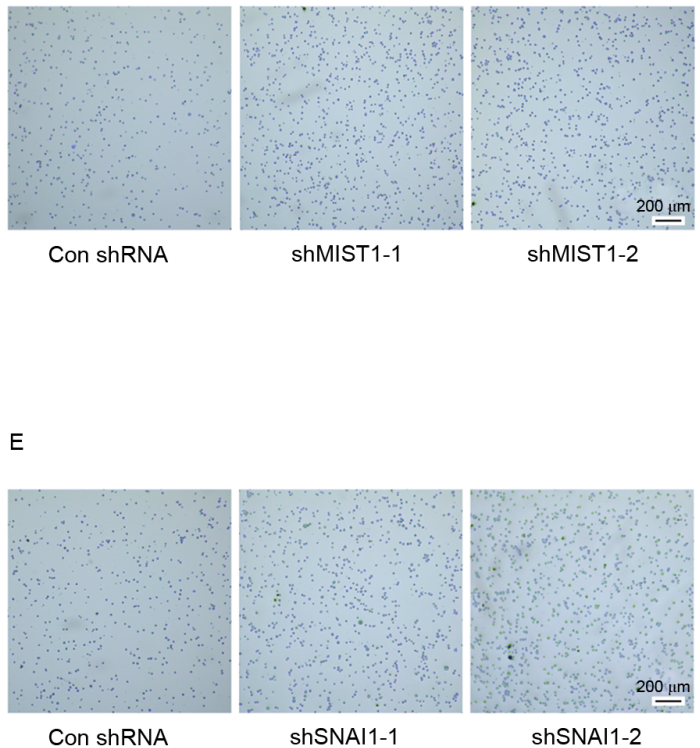

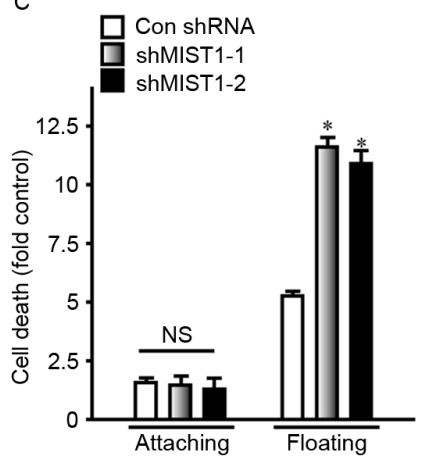

F

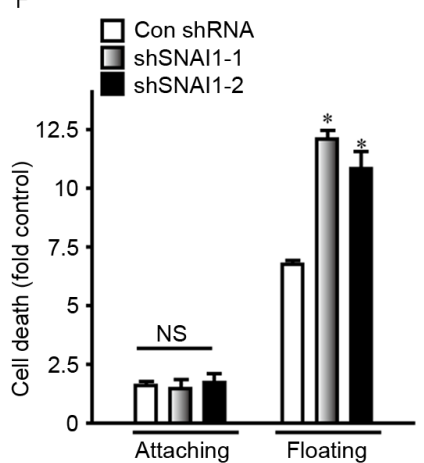

Figure 3. Knockdown of MIST1 and SNAI1 increases adhesion and induces cells to undergo anoikis. (A) MIST1 was knocked down in the A375 and MV3 cell lines, as confirmed by western blot analysis. (B) Knockdown of MIST1 improved the adhesion ability of A375 cells (scale bar, $200 \mu$ m). Findings for MV3 were similar, data not shown. (C) Inhibition of MIST1 decreased the viability of A375 cells in suspension culture, indicating increased anoikis. Findings for MV3 were similar, data not shown. (D) SNAI1 was knocked down in the A375 and MV3 cell lines, as confirmed by western blot analysis. (E) Knockdown of SNAI1 improved the adhesion ability of A375 cells (scale bar, $200 \mu \mathrm{m}$ ). (F) Inhibition of SNAI1 decreased the viability of A375 cells in suspension culture, indicating increased anoikis. NS, no significance; ${ }^{\mathrm{P}}<0.05$ vs. control. shMIST1, small hairpin RNA targeting muscle intestine and stomach expression 1 ; Con, control.

Following loss of anchorage, MIST1 and SNAI1 protected cells from anoikis.

Knockdown of MIST1 and SNAII increases adhesion and induces anoikis in melanoma cells. Conversely, to determine the role of MIST1 and SNAI1 in human melanoma cells, their expression was altered in tumor cell lines. shRNAs were utilized to reduce the expression of MIST1 and SNAI1 in the A375 and MV3 human melanoma cell lines (Fig. 3). In order to avoid off-target effects, two target sequences were used to reduce expression of MIST1 and SNAI1, respectively, namely vector-shMIST1-1, vector-shMIST1-2, vector-shSNAI1-1 and vector-shSNAI1-2. The knockdown of MIST1 was detected by immunoblot (Fig. 3A). A fibronectin adhesion assay was also performed to confirm the influence of MIST1 on the attachment to the ECM. Knockdown of MIST1 increased the binding of A375 and MV3 human melanoma cell lines to fibronectin (Fig. 3B). After suspension culture for $24 \mathrm{~h}$, the apoptotic rate of A375 and MV3 cells was significantly increased following MIST1 knockdown (Fig. 3C). Furthermore, knockdown of SNAI1 was revealed to have a similar effect to that of MIST1 (Fig. 3D-F). It was apparent that knockdown of endogenous MIST1 and SNAI1 inhibited anchorage independence of human melanoma cells and increased their sensitivity to anoikis. The results of the gain- and loss-of-function studies suggested that MIST1 and SNAI1 help human melanoma cells to part from the ECM and bypass anoikis, which may contribute to the formation of distant metastases.
MIST1 confers anoikis resistance through regulating SNAI1. As the abovementioned results indicated that MIST1 and SNAI1 had a role in the development and progression of human melanoma cells, the association between them was then assessed. To define the correlation between MIST1 and SNAI1, these two genes were ectopically overexpressed in HUVEC and NHEM cells, which do not highly express them. The results demonstrated that only MIST1 overexpression caused an increase in the expression of SNAI1, but not vice versa (Fig. 4A). After enhancement of the expression of MIST1 or SNAI1, the resistance to anoikis was obviously increased (Fig. 4B). Conversely, knockdown of MIST1 decreased the expression of SNAI1 in the human melanoma cell lines A375 and MV3, which express the two genes at relatively high levels, while knockdown of SNAI1 did not affect the levels of MIST1 (Fig. 4C). After knockdown of MIST1 or SNAI1, the resistance to anoikis was obviously decreased (Fig. 4D). As alteration of MIST1 caused a change of SNAIL1 but not vice versa, it was indicated that MIST1 is an upstream gene of SNAIL1. Furthermore, the results indicated that MIST1 promotes anchorage independence of cells through regulating SNAI1.

MIST1 hijacks the PTEN/AKT signaling pathway via SNAI1. To investigate the molecular mechanisms via which MIST1 and SNAI1, which are highly expressed in the A375 and MV3 human melanoma cell lines, reduce their sensitivity to anoikis, the activation of Akt kinase, the downstream substrate of PI3K, was examined. Dysregulation of Akt is 


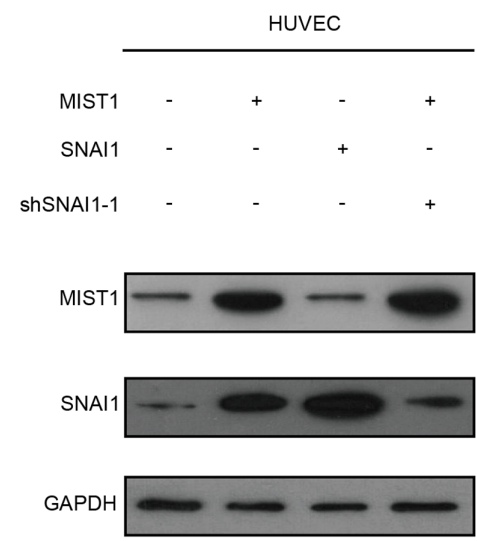

C

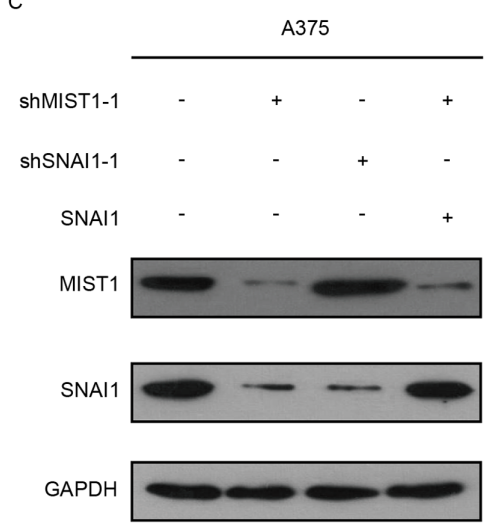

B

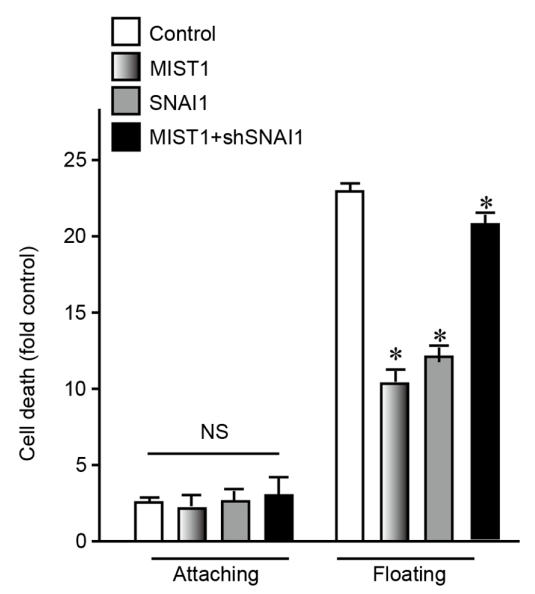

$\mathrm{D}$

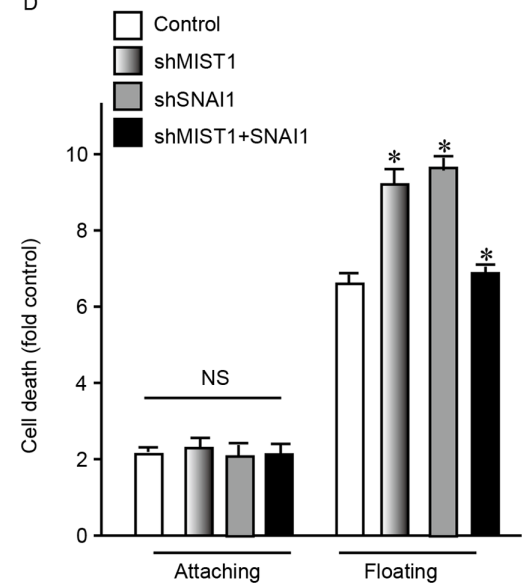

Figure 4. MIST1 confers anoikis resistance through regulating SNAI1. (A and B) HUVEC were transduced with MIST1 or SNAI1 overexpression vector, or with MIST1 overexpression in combination with shSNAI1. (A) Confirmation of protein expression by western blot analysis. (B) The rate of cell death of attached and floating cells was assessed after $24 \mathrm{~h}$. (C and D) A375s were transduced with shMIST1-1 or shSNAI1-1, or with shMIST1-1 in combination with SNAI1 expression plasmid. (C) Confirmation of protein expression by western blot analysis. (D) The rate of cell death of attached and floating cells was assessed after $24 \mathrm{~h}$. SNAI1 was positively associated with changes of MIST1 in HUVEC and A375 cells. Findings for NHEM 2493 were similar with HUVEC and findings for MV3 were similar with A375, data not shown. NS, no significance; ${ }^{*} \mathrm{P}<0.05$ vs. control. shMIST1, small hairpin RNA targeting muscle intestine and stomach expression 1; HUVEC, human umbilical vein endothelial cells.

commonly found in tumor cells and has an important role in resistance to anoikis and promoting cell survival (25). AKT phosphorylation at the Ser473 residue was obviously increased following MIST1 overexpression in the normal human HUVEC cell line. In addition, it was previously reported that SNAI1 directly repressed PTEN, which then led to PI3K/Akt pathway activation, thus contributing to anoikis resistance (23). Consistent with these previous findings, the present study discovered downregulation of PTEN by MIST1 and SNAI1 (Fig. 5A). As a competitive inhibitor of PI3K, LY294002 is generally used to suppress p-AKT (Ser473), and was applied in the present study at $10 \mu \mathrm{M}$ to reduce $\mathrm{p}$-AKT (Ser473). After inhibition of AKT phosphorylation (Ser473), the MIST1-induced reduction in sensitivity to anoikis was restored (Fig. 5A and B).

The expression of PTEN was increased in the A375 and MV3 human melanoma cell lines after knockdown of MIST-1. Correspondingly, the levels of p-AKT (Ser473) were obviously decreased (Fig. 5C). shRNA targeting PTEN was used to reduce its expression, which abrogated the decrease in the levels of p-AKT (Ser473) caused by MIST1 knockdown (Fig. 5C). In addition, the MIST1 knockdown-associated increases in anoikis were inhibited by simultaneous PTEN knockdown.

The results of the gain- and loss-of-function study suggested that the PTEN/Akt signaling pathway is involved in the mechanism by which MIST1 and SNAI1 mediat anoikis resistance in human melanoma cells.

MIST1 promotes SNAI1 transcription by directly binding to its promoter region. The aforementioned results confirmed that MIST1 stimulatestheexpression of SNAI1, butthespecificmechanism required further exploration. The MIST1 binding motif predicted by the JASPAR database (http://jaspar.genereg.net/) exists in a binding sequence predicted by the ENCODE database (https://genome.ucsc.edu/ENCODE/) (Fig. 6A). ChIP was used to confirm that MIST1 directly regulated SNAI1. For this experiment, seven regions close to the transcription start site were selected (Fig. 6B). Protein G PLUS-Agarose binding with MIST1 antibody was used to pull down DNA-binding fragments. A ChIP assay using HUVEC and NHEM overexpressing MIST1, the amplification intensity of the R5 fragment from the SNAI1 promoter was significantly higher than that of the other DNA segments, as confirmed by 


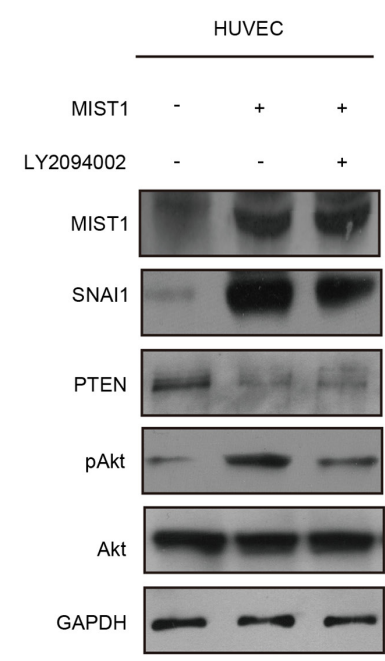

$\mathrm{C}$

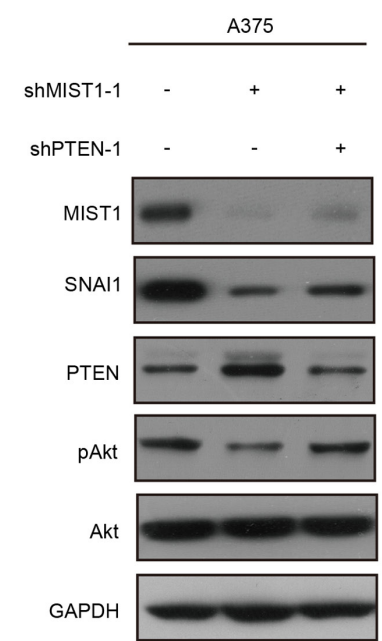

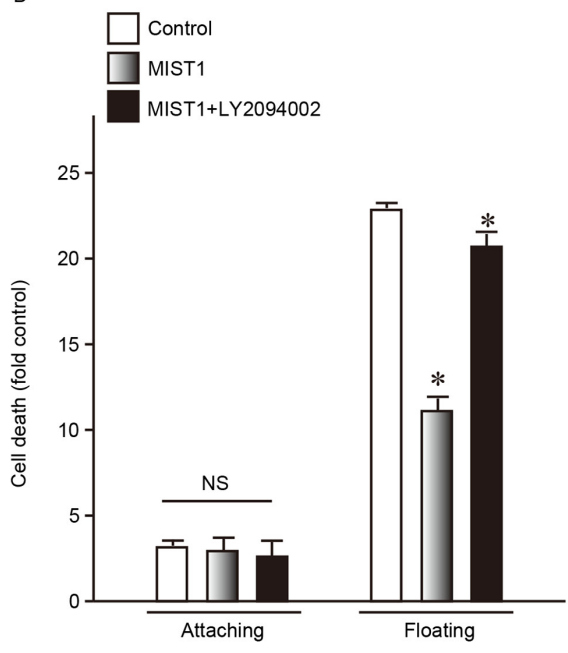

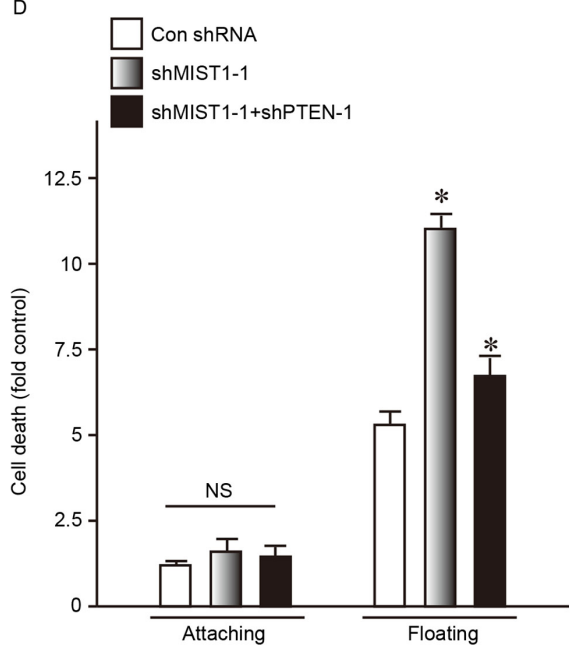

Figure 5. MIST1 hijacks the PTEN/AKT signaling pathway via SNAI1. (A and B) Western blot analysis indicated that overexpression of MIST1 reduced the expression of PTEN through SNAI1 and further increased p-AKT (S473) in HUVEC. Inhibitor of p-AKT (S473) LY294002 restored this change and the ability to resist anoikis. (C and D) Knockdown of MIST1 increased the expression of PTEN through SNAI1 and further reduced p-AKT (S473) in A375, as indicated by western blot analysis. Knockdown of PTEN increased p-AKT (S473) and promoted anchorage independence. ${ }^{\text {P }}<0.05$ vs. control. shMIST1, small hairpin RNA targeting muscle intestine and stomach expression 1; HUVEC, human umbilical vein endothelial cells; p-PTEN, phosphorylated phosphatase and tensin homologue. Findings for MV3 were similar with A375, data not shown.

PCR (Fig. 6C and D). A375 and MV3, which highly express MIST1, were transfected with luciferase reporter vectors. Vectors containing the $-1,199$ to -753 bp fragment demonstrated enhanced luciferase reporter signaling (Fig. 6E). Taken together, these results strongly indicated that MIST1 regulates the expression of SNAI1 through binding to its promoter to ultimately confer resistance to anoikis in human melanoma cells.

\section{Discussion}

Melanoma is a melanocytic malignancy derived from the pigmented areas of the skin, mucous membrane, eye and central nervous system, with a high degree of malignancy and a poor prognosis. According to statistics, $~ 160,000$ patients are newly diagnosed with melanoma worldwide and $\sim 48,000$ succumb to melanoma each year (26). The 5-year survival rate of patients with early, advanced and late-stage melanoma, respectively, was $91.2,61.7$ and $15.2 \%$. In addition, the recurrence rate in melanoma patients in remission is 9 times that of other cancer types (26). Current treatments used for melanoma are surgery, radiation therapy, chemotherapy and immunotherapy. The clinical application of the cytotoxic T-lymphocyte-associated protein 4 antibody ipilimumab and the BRAF inhibitor vemurafenib, which were approved for the treatment of advanced melanoma and BRAF mutant melanoma in 2011, improved the treatment of melanoma to a certain degree, a requirement for more in-depth study of the mechanisms underlying the development and metastasis of melanoma remains, as more effective targets to combat melanoma require to be developed.

The present study focused on the key step in the metastasis of melanoma, namely resistance to anoikis. Anoikis helps tumor cells to survive during migration from the original matrix into the blood and lymph nodes. Studies have indicated that ECM as an inhibitor of anoikis and integrins has a profound effect on cell survival. The most important factors in integrin-regulated signal transduction pathways include 

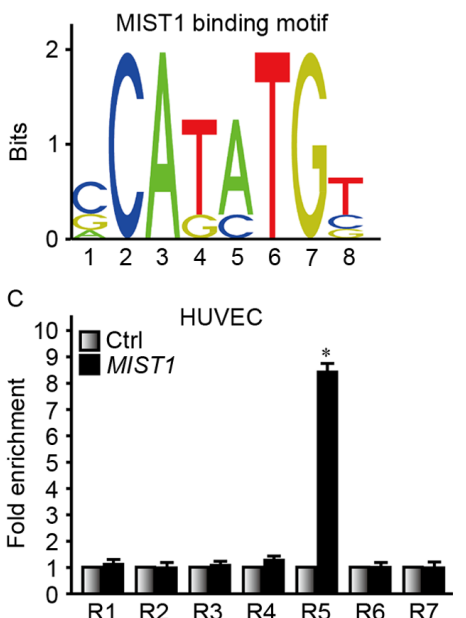
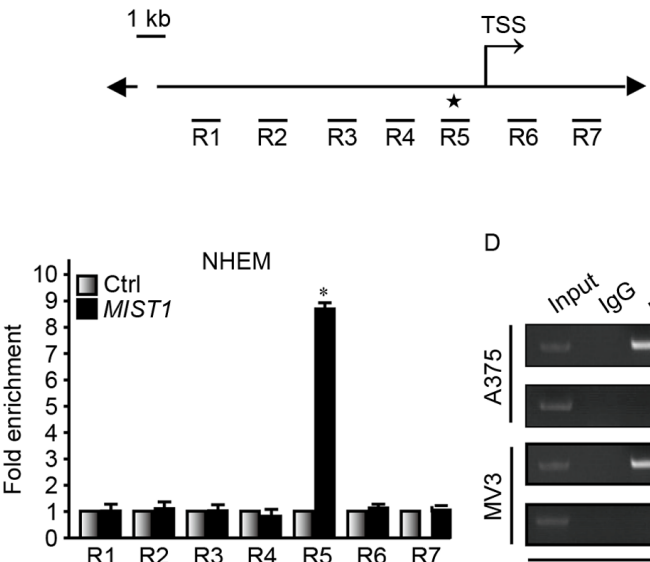

$\stackrel{\text { TSS }}{\longrightarrow}$

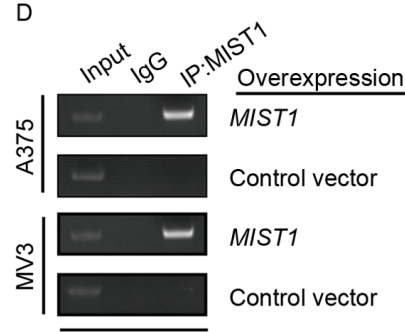

R5

$\mathrm{E}$
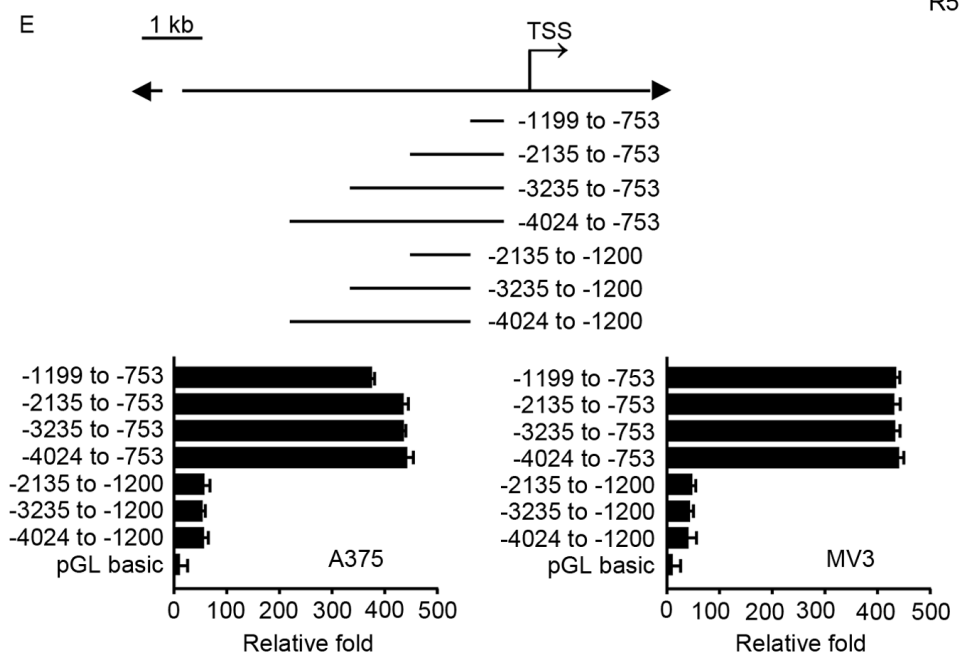

Figure 6. MIST1 promotes SNAI1 transcription by directly binding to its promoter region. (A) MIST1 binding motif. (B) Design of 7 chromatin regions of the SNAI1 promoter containing the MIST1 binding motif for ChIP. (C and D) ChIP performed on HUVEC and NHEM 2493 cells demonstrated that MIST1 was enriched in samples containing region 5 of the SNAI1 promoter. (E) A luciferase reporter assay determined that the -1,199 to -753 bp segment upstream of TSS site of SNAI1 combined with MIST1. TSS, transcription start site; Ctrl, control; IgG, immunoglobulin G; ChIP, chromatin immunoprecipitation; * $<<0.05$ vs. control; MIST1, muscle intestine and stomach expression 1; HUVEC, human umbilical vein endothelial cells; NHEM 2493, normal human epidermal melanocytes.

focal adhesion kinase, integrin-linked protein kinase, tyrosine kinase, PI3K, extracellular signal-regulated kinase and connexin Shc (27). The present study elucidated the upstream signaling of the PTEN/Akt signaling pathway with regard to anoikis.

MIST1 exists in numerous tissue types, but regarding the cell type, mainly in serous exocrine cells. MIST1 is the first transcription factor identified to be a protein specifically expressed by serous exocrine cells and has a key role in the formation and maintenance of serous secretory exocytosis. As a well-known transcription factor, SNAI1 has a critical role in EMT of tumor cells. However, the association between MIST1 and SNAI1 and their role in tumor progression has remained to be elucidated.

In the present study, MIST1 was found to be upregulated in the A375 and MV3 human melanoma cell lines. Ectopic overexpression of MIST1 increased the resistance of normal cells to anoikis. Conversely, knockdown of MIST1 expression reduced the resistance of melanoma cells to anoikis. These suggested that MIST1 has a critical role in the resistance of melanoma cells to anoikis. Gain- and loss-of-function experiments and ChIP confirmed that SNAI1 was the downstream gene of MIST1. Furthermore, a luciferase reporter assay located the MIST1 recognition sequence at $-1,199$ to $-753 \mathrm{bp}$ of the SNAI1 gene promoter. It was further demonstrated that MIST1 promotes the evasion of anoikis by melanoma cells through the PTEN/Akt pathway.

To the best of our knowledge, the present study was the first to report that MIST1 binds directly to the promoter region of SNAI1 and regulates its expression. It was previously demonstrated that SNAI1 inhibits the phosphorylation of Akt by inhibiting the expression of PTEN (23). The present study provided an update regarding the upstream signaling of the PTEN/Akt pathways. This provided a novel target for the clinical treatment of melanoma. However, the specific mechanism underlying the upregulation of MIST1 in melanoma has remained elusive, and its study may provide an approach for the clinical treatment of melanoma. In addition, the role of MIST1 and SNAI1 in anoikis resistance requires further verification in a wide range of tumor types. 


\section{References}

1. Jemal A, Siegel R, Ward E, Hao Y, Xu J and Thun MJ: Cancer statistics, 2009. CA Cancer J Clin 59: 225-249, 2009.

2. High WA and Robinson WA: Genetic mutations involved in melanoma: A summary of our current understanding. Adv Dermatol 23: 61-79, 2007.

3. Giancotti FG and Tarone G: Positional control of cell fate through joint integrin/receptor protein kinase signaling. Annu Rev Cell Dev Biol 19: 173-206, 2003.

4. Paoli P, Giannoni E and Chiarugi P: Anoikis molecular pathways and its role in cancer progression. Biochim Biophys Acta 1833: 3481-3498, 2013.

5. Tan K, Goldstein D, Crowe P and Yang JL: Uncovering a key to the process of metastasis in human cancers: A review of critical regulators of anoikis. J Cancer Res Clin Oncol 139: 1795-1805, 2013.

6. Yang J, Zheng Z, Yan X, Li X, Liu Z and Ma Z: Integration of autophagy and anoikis resistance in solid tumors. Anat Rec (Hoboken) 296: 1501-1508, 2013.

7. Thompson EW, Newgreen DF and Tarin D: Carcinoma invasion and metastasis: A role for epithelial-mesenchymal transition? Cancer Res 65: 5991-5995, 2005.

8. Zanotti S, Gibertini S, Bragato C, Mantegazza R, Morandi L and Mora M: Fibroblasts from the muscles of Duchenne muscular dystrophy patients are resistant to cell detachment apoptosis. Exp Cell Res 317: 2536-2547, 2011.

9. Kadesch T: Consequences of heteromeric interactions among helix-loop-helix proteins. Cell Growth Differ 4: 49-55, 1993.

10. Jia D, Sun Y and Konieczny SF: Mist1 regulates pancreatic acinar cell proliferation through p21 CIP1/WAF1. Gastroenterology 135: $1687-1697,2008$.

11. Wakamatsu Y, Sun Y and Konieczny SF: Comparative gene expression analyses reveal heterochrony for Sox 9 expression in the cranial neural crest during marsupial development. Evol Dev 16: 197-206, 2014.

12. Wu ZQ, Rowe RG, Lim KC, Lin Y, Willis A, Tang Y, Li XY, Nor JE, Maillard I and Weiss SJ: A Snail1/Notch1 signalling axis controls embryonic vascular development. Nat Commun 5: 3998 2014.

13. Usova EV, Kopantseva MR, Egorov VI, Kopantzev EP and Sverdlov ED: SNAI1 and SNAI2-transcriptional master-regulators of epithelial-mesenchimal transition). Patol Fiziol Eksp Ter 59: 76-87, 2015 (In Russian).

14. Herranz N, Pasini D, Díaz VM, Francí C, Gutierrez A, Dave N, Escrivà M, Hernandez-Muñoz I, Di Croce L, Helin K, et al: Polycomb complex 2 is required for E-cadherin repression by the Snaill transcription factor. Mol Cell Biol 28: 4772-4781, 2008.

15. Moreno-Bueno G, Peinado H, Molina P, Olmeda D, Cubillo E, Santos V, Palacios J, Portillo F and Cano A: The morphological and molecular features of the epithelial-to-mesenchymal transition. Nat Protoc 4: 1591-1613, 2009.
16. Wu Y and Zhou BP: Snail: More than EMT. Cell Adh Migr 4: 199-203, 2010

17. Escrivà $\mathrm{M}$, Peiró $\mathrm{S}$, Herranz $\mathrm{N}$, Villagrasa $\mathrm{P}$, Dave $\mathrm{N}$, Montserrat-Sentís B, Murray SA, Francí C, Gridley T, Virtanen I and García de Herreros A: Repression of PTEN phosphatase by Snaill transcriptional factor during gamma radiation-induced apoptosis. Mol Cell Biol 28: 1528-1540, 2008.

18. Zhang L, Wu J, Ling MT, Zhao L and Zhao KN: The role of the $\mathrm{PI} 3 \mathrm{~K} / \mathrm{Akt} / \mathrm{mTOR}$ signalling pathway in human cancers induced by infection with human papillomaviruses. Mol Cancer 14: 87, 2015.

19. Guidetti GF, Canobbio I and Torti M: PI3K/Akt in platelet integrin signaling and implications in thrombosis. Adv Biol Regul 59: 36-52, 2015

20. Fokas E, McKenna WG and Muschel RJ: The impact of tumor microenvironment on cancer treatment and its modulation by direct and indirect antivascular strategies. Cancer Metastasis Rev 31: 823-842, 2012.

21. Safdari Y, Khalili M, Ebrahimzadeh MA, Yazdani Y and Farajnia S: Natural inhibitors of PI3K/AKT signaling in breast cancer: Emphasis on newly-discovered molecular mechanisms of action. Pharmacol Res 93: 1-10, 2015.

22. Chung JH and Eng C: Nuclear-cytoplasmic partitioning of phosphatase and tensin homologue deleted on chromosome 10 (PTEN) differentially regulates the cell cycle and apoptosis. Cancer Res 65: 8096-8100, 2005.

23. Barrallo-Gimeno A and Nieto MA: The Snail genes as inducers of cell movement and survival: Implications in development and cancer. Development 132: 3151-3161, 2005.

24. Pin CL, Bonvissuto AC and Konieczny SF: Mist1 expression is a common link among serous exocrine cells exhibiting regulated exocytosis. Anat Rec 259: 157-167, 2000.

25. Sheng S, Qiao M and Pardee AB: Metastasis and AKT activation. J Cell Physiol 218: 451-454, 2009.

26. Lozano R, Naghavi M, Foreman K, Lim S, Shibuya K, Aboyans V, Abraham J, Adair T, Aggarwal R, Ahn SY, et al: Global and regional mortality from 235 causes of death for 20 age groups in 1990 and 2010: A systematic analysis for the Global Burden of Disease Study 2010. Lancet 380: 2095-2128, 2012.

27. Hanks SK, Ryzhova L, Shin NY and Brábek J: Focal adhesion kinase signaling activities and their implications in the control of cell survival and motility. Front Biosci 8: d982-d996, 2003.

This work is licensed under a Creative Commons Attribution-NonCommercial-NoDerivatives 4.0 International (CC BY-NC-ND 4.0) License. 